\title{
The Relation of Angiographic-Based Coronary Artery Diameters with Gender and Traditional Cardiovascular Risk Factors in Patients with Normal or Mild CAD
}

\author{
Farshad Shakerian, ${ }^{1}$ Yaghoob Bagheri, ${ }^{1}$ Sara Shemshadi, ${ }^{1}$ Ata Firouzi, ${ }^{1}$ Hamid Reza Sanati, ${ }^{1,}$ Reza \\ Kiani, ${ }^{1}$ Ali Zahedmehr, ${ }^{1}$ Parham Sadeghipour, ${ }^{1}$ and Reza Bakhtiari ${ }^{1}$ \\ ${ }^{1}$ Cardiovascular Intervention Research Center, Rajaie Cardiovascular Medical and Research Center, Iran University of Medical Sciences, Tehran, IR Iran \\ "Corresponding author: Hamid Reza Sanati, Department of Interventional Cardiology, Rajaie Cardiovascular Medical and Research Center, Vali-Asr St., Niayesh Blvd, Tehran, IR \\ Iran. Tel: +98-2123922177, +98-9123765828, E-mail: sanati56@yahoo.com
}

Received 2016 April 16; Revised 2016 August 05; Accepted 2016 August 24.

\begin{abstract}
Background: Several physiological and pathological processes affect the diameter of coronary arteries. It seems that the pathological increase or decrease in the size of coronary arteries is associated with cardiovascular events.

Objectives: The aim of this study is to determine the relationship between the diameter of coronary arteries, gender, and traditional cardiovascular risk factors.

Methods: In this cross-sectional study, we enrolled 96 patients who underwent coronary angiography at our tertiary research center. The patients' demographic data, cardiovascular risk factors, and coronary artery diameters were recorded using a digital sliding caliper.

Results: Out of 96 patients with a mean age of $56.3 \pm 9.8$ years, 62 (64.6\%) were female. Among cardiovascular risk factors dyslipidemia was the most prevalent (55 patients or 57.3\%) followed by hypertension (45 patients or $46.9 \%$ ) and smoking (17 patients or 17.7\%). The means of the right coronary artery (RCA), left main coronary artery (LMCA), left anterior descending (LAD) and left circumflex (LCX) diameters were $3.27 \pm 0.7 \mathrm{~mm}, 4.35 \pm 0.7 \mathrm{~mm}, 3.5 \pm 0.6 \mathrm{~mm}$ and $3 \pm 0.7 \mathrm{~mm}$, respectively. The female and participants and participants with diabetes had smaller coronary arteries: these differences have become statistically significant for LMCA and LAD in both groups (in women, the p value for LMCA and LAD were 0.04 and 0.02 , respectively, and in those with diabetics, the $\mathrm{p}$ value for LMCA and LAD were 0.02 and 0.04 , respectively).

Conclusions: In our study, female participants and participants with diabetes have smaller coronary arteries. No statistically significant relationships were found between traditional coronary risk factors and the diameters of coronary arteries.
\end{abstract}

Keywords: Selective Coronary Angiography, Coronary Artery Diameter, Diabetes Mellitus, Traditional Coronary Risk Factors

\section{Background}

The pathological impact of coronary vessel size has been the subject of debate. We have some evidence that coronary artery size matters and both pathological decrease and increase in the size of coronary vasculature might lead to an adverse cardiovascular outcome. First, through epidemiological studies, researchers have found that women have worse coronary artery disease outcomes than men. Several explanations have been proposed with smaller coronary artery caliber in female populations being among them. Apart from that we have learnt from coronary stenting studies that all the major adverse events related to stenting (e.g. in-stent restenosis, stent thrombosis and etc.) occurred more frequently in patients with smaller coronary arteries. Also, in patients who underwent coronary bypass graft surgery (CABG), the graft patency had a direct relationship to the vessel size. Finally, thrombotic occlusions have occurred more frequently during plaque rupture in small caliber coronary arteries.

While earlier studies showed the importance of the reduction in coronary artery diameter in pathological states, recent investigation on the evolution of vulnerable plaques has shown the importance of positive remodeling. These works have observed that vessels with a larger cross-sectional area due to positive remodeling are more vulnerable to plaque rupture. Aside from this phenomena, a pathological increase in the size of coronary arteries such as coronary artery ectasia or an aneurysm has been linked to adverse outcomes.

The challenge for finding factors which influence the size of coronary arteries started long ago. Early studies commonly investigated the relation of gender, heart weight, and age with the size of coronary arteries. Among 
the traditional risk factors, hypertension with its effect on heart weight via the hypertrophic remodeling might have an effect on the size of coronary arteries. The relationship between other traditional risk factors and coronary artery diameter have been less frequently studied. Traditional risk factors that increase the atherosclerotic burden could influence the progression of atherosclerotic plaque resulting in positive remodeling and increasing the cross-sectional area. Finally, the effect of diabetes mellitus (DM) on the coronary vasculature have been widely investigated. We know now that people with diabetes have poorer cardiovascular outcomes than the general population. One of the factors which have been suggested as a contributor to this poor outcome is the influence of DM on vessel caliber.

\section{Objectives}

For the present study, we used selective coronary angiography to examine the effect of gender, DM, and traditional risk factors on the coronary arteries diameter.

\section{Methods}

\subsection{Patients and Method}

In this prospective cross-sectional study, all patients referred to our tertiary research center were selected by a simple sampling method. Among those who have undergone angiography, patients with normal angiograms and mild coronary artery disease (with a minimal lumen diameter of less than $50 \%$ of the reference lumen diameter) were included. The exclusion criteria were patients diagnosed with variant angina, a history of acute coronary syndrome, a history of revascularization, end-stage renal disease, and an allergy to the contrast media. All patients have signed the informed consent. All the demographic, laboratory, and clinical information for each patient was collected from the center's database.

Selective coronary angiographies were performed by the Judkins technique, using either a femoral or radial artery approach. For each patient, we obtained all the classic angiographic projections, and further projections were taken as needed. The diameters were recorded using a digital sliding caliper (Mitutoyo Corporation, Japan) capable of measuring to the nearest $0.01 \mathrm{~mm}$. All the measurements were repeated three times by an interventional cardiologist, and the mean was taken for further analysis.

\subsection{Statistical Analysis}

The data distribution was checked by the One Sample Kolmogorov-Smirnov Test. Results were expressed as mean \pm SD. All the data were analyzed using IBM SPSS Statistics 19 for Windows (IBM Corporation, Armonk, NY, USA). The Mann-Whitney U test or Student's t-test was used to compare the relationship between the diameter of the coronary vessels and the CAD risk factor. With regards to adjusting or removing the confounding factor effects, Multiple Logistic Regression Analyses were used. A P value $<0.05$ was considered statistically significant. Post-hoc Power was calculated to evaluate the association between coronary artery diameter and relevant risk factors.

\section{Results}

In the present study, 96 patients (34 or $35.4 \%$ male with a mean age of $56.3 \pm 9.8$ ) were enrolled between August 2014 and early October 2015. Patients' demographic data are depicted in Table1. In our evaluation, the most frequent risk factor was dyslipidemia observed in 55 patients (57.3\%) followed by hypertension in 45 patients (49.6\%), and smoking in 17 patients (17.7\%). The mean artery diameter for each vessel was also reported in Table 1.

Table 1. All Patients' Demographic and Baseline Data

\begin{tabular}{|cc|}
\hline Variables & Values $^{\mathbf{a}}$ \\
\hline Sex $(\mathbf{F} / \mathbf{M})$ & $62(64.6) / 34(35.4)$ \\
\hline Age & $56.3 \pm 9.8$ \\
\hline Risk Factors & $27(28.1)$ \\
\hline DM & $45(46.9)$ \\
\hline HTN & $55(57.3)$ \\
\hline DLP & $17(17.7)$ \\
\hline C/S & $15(15.6)$ \\
\hline FH & $28 \pm 4.7$ \\
\hline BMI & \\
\hline Coronary artery diameter & $3.5 \pm 0.6$ \\
\hline LAD & $3 \pm 0.7$ \\
\hline LCX & $3.27 \pm 0.7$ \\
\hline RCA & $4.35 \pm 0.7$ \\
\hline LMCA & \\
\hline
\end{tabular}

${ }^{\mathrm{a}}$ Values are expressed as mean $\pm \mathrm{SD}$ or No. (\%).

The relationship between coronary arteries diameter with gender and cardiovascular risk factors are shown in Table 2. Age was not an influential determinant of coronary artery diameter $(\mathrm{P}$ value $=0.1)$. In our study, women 
tended to have smaller coronary arteries. But this difference became statistically significant only in LMCA and LAD ( $\mathrm{P}$ value $=0.04, \mathrm{P}$ value $=0.02$, respectively $)$. There were no significant differences regarding LMCA, LAD, LCX, and RCA, between the two groups with and without dyslipidemia ( $P$ value $=0.4$, P value $=0.4, \mathrm{P}$ value $=0.2, \mathrm{P}$ value $=0.5$; Table 2). Other risk factors such as hypertension, smoking, and familial history, did not have an influence on the vessel size (Table 2). The trend in our population with diabetes was a smaller coronary artery diameter compared to participants without diabetes, but, like gender, this difference was only statistically significant for the LMCA and LAD (P value $=0.02$ and $\mathrm{P}$ value $=0.04$, respectively; Table 2 ) .

Multivariate analysis was used to remove the confounding factor effects, and the association between those variables and outcome were assessed. Among all the variables, the association of age, sex, dyslipidemia, DM, familial history, cigarette smoking, and HTN as confounding factors and outcome were assessed, and the results showed that only sex was significantly related to LMCA diameter ( $P$ value $=0.02$; Table 3 )

\section{Discussion}

This study was performed to assess the correlation between coronary artery dimensions and gender and cardiovascular risk factors. We did not find any relationship between the traditional risk factors and coronary artery size. Age was also not a determinant. However, our female population and participants with diabetes have smaller coronary arteries.

The importance of anthropological factors on cardiovascular outcome has been the subject of multiple studies. Coronary artery diameter as a determinant has been well-studied. Earlier works focused on small coronary arteries and their impact on the cardiovascular outcome. There were some epidemiologic observations for this hypothesis. First, women have worse prognoses following both acute coronary syndrome events and revascularization. Several explanations have been suggested for this phenomena with the smaller caliber of coronary artery among them (1-3). Also, the minimal lumen diameter has been related to various adverse events following percutaneous coronary intervention (PCI); restenosis and stent thrombosis occurred more frequently in smaller coronary vessels (4). The same story has also been observed during CABG; grafts being anastomosed to smaller arteries have a lower patency rate (5). Finally, thrombotic occlusion followed by plaque rupture are more frequent in small arteries (6). Apart from the influence of small coronary arteries on cardiovascular outcomes, emerging evidence has shown that vessels enlarged by positive remodeling have a greater atherosclerotic plaque burden and are more vulnerable $(7,8)$.

Several anthropological and clinical factors have been suggested as influential factors on coronary artery diameter. In our study, the female participants had smaller coronary arteries. Statistically, LMCA and LAD (with P value = 0.02 and 0.04 , respectively were significantly smaller in women compared to men. Surprisingly, age was not a significant determinant in the population we studied. Both age and gender have been well-studied as factors influencing the coronary artery diameter, and several studies have proved this impact (9-12). However, it is still unclear whether the correlation between gender and the size of the coronary artery is simply because of smaller body surface area or if it has an independent effect $(10,11,13,14)$. The same is true for age; some studies did not find a reproducible relationship between age and the size of a coronary artery (15).

In our study, none of the traditional cardiac risk factors have a relationship with coronary artery size. In one the few studies on this issue, Hamirani et al. measured the diameter of the coronary arteries of 140 patients with 64slice multidetector computed tomography and reported its relationship with traditional risk factors (16). In this study, patients with a larger RCA were more hypertensive. However, no positive relationships with the size of coronary arteries and other risk factors were detected (16). McGill et al. in a postmortem study of 2,403 individuals, found that hypertensive participants have larger RCA and LMCA (17).

There is now solid evidence that DM affects the anatomy and structure of coronary arteries (18). Mosseri et al. in their analysis of 711 patients with angiographically normal coronary arteries, concluded that coronary arteries and their branches are smaller among people with diabetes (19). Our patients with DM also have a smaller coronary artery diameter, and we have observed a significant difference in the size of LMCA and LAD compared to the population without DM.

\subsection{Conclusions}

In the present study, we evaluate the potential relationship of coronary artery size, gender, and traditional risk factors. Among all the studied determinants, only gender and DM have a statistical relation with coronary artery size. Apart from the contribution to a better understanding of coronary artery disease, the matter of coronary artery diameter has a practical implication in the area of revascularization. By knowing the influential factors and their specific impact on coronary artery diameter, a more durable strategy for revascularization can be chosen. Obviously, 
Table 2. Coronary Arteries Diameter Relationship with Cardiovascular Risk Factors

\begin{tabular}{|c|c|c|c|c|c|c|c|c|c|}
\hline & & LAD & P Value & LCX & PValue & RCA & P Value & LMCA & P Value \\
\hline Sex & & & 0.02 & & 0.5 & & 0.36 & & 0.04 \\
\hline & Female & $3.4 \pm 0.6$ & & $3.05 \pm 0.7$ & & $3.2 \pm 0.7$ & & $4.2 \pm 0.8$ & \\
\hline & Male & $3.7 \pm 0.6$ & & $3.1 \pm 0.8$ & & $3.3 \pm 0.8$ & & $4.5 \pm 0.6$ & \\
\hline DM & & & 0.04 & & 0.6 & & 0.36 & & 0.02 \\
\hline & + & $3.3 \pm 0.47$ & & $3 \pm 0.69$ & & $3.1 \pm 0.7$ & & $4 \pm 0.69$ & \\
\hline & - & $3.58 \pm 0.6$ & & $3.1 \pm 0.8$ & & $3.3 \pm 0.78$ & & $4.4 \pm 0.78$ & \\
\hline DLP & & & 0.4 & & 0.4 & & 0.28 & & 0.59 \\
\hline & + & $3.5 \pm 0.6$ & & $3.1 \pm 0.8$ & & $3.1 \pm 0.8$ & & $4.38 \pm 0.8$ & \\
\hline & - & $3.4 \pm 0.58$ & & $3.02 \pm 0.7$ & & $3.3 \pm 0.7$ & & $4.3 \pm 0.7$ & \\
\hline HTN & 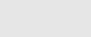 & & 0.8 & & 0.5 & & 0.4 & & 0.8 \\
\hline & + & $3.5 \pm 0.6$ & & $3.1 \pm 0.7$ & & $3.3 \pm 0.8$ & & $4.37 \pm 0.7$ & \\
\hline & - & $3.5 \pm 0.6$ & & $3 \pm 0.8$ & & $3.2 \pm 0.68$ & & $4.3 \pm 0.79$ & \\
\hline $\mathrm{C} / \mathrm{S}$ & & & 0.1 & & 0.7 & & 0.19 & & 0.5 \\
\hline & + & $3.7 \pm 0.67$ & & $3.1 \pm 0.8$ & & $3.5 \pm 0.7$ & & $4.4 \pm 0.7$ & \\
\hline & - & $3.4 \pm 0.6$ & & $3.08 \pm 0.7$ & & $3.2 \pm 0.78$ & & $4.3 \pm 0.78$ & \\
\hline FH & & & 0.1 & & 0.3 & & 0.5 & & 0.6 \\
\hline & + & $3.7 \pm 0.68$ & & $3.2 \pm 0.8$ & & $3.38 \pm 0.89$ & & $4.4 \pm 0.89$ & \\
\hline & - & $3.4 \pm 0.6$ & & $3.06 \pm 0.7$ & & $3.2 \pm 0.7$ & & $4.3 \pm 0.7$ & \\
\hline
\end{tabular}

Abbreviations: C/S, cigar smoking; DLP, dyslipidemia; DM, diabetes mellitus; FH, family history; HTN, hypertension; LAD, left anterior descending, LCX, left circumflex artery; LMCA, left main coronary artery; RCA, right coronary artery.

our results and all the mentioned studies agree on the importance of coronary artery diameter and its impact on the prognosis of the patient. But we still do not know whether the diameter is inherited or is just influenced by environmental factors. Does our traditional effort for primary and secondary prevention impact this index or we need some new strategies? High-quality observational studies with an acceptable population will clear the matter. In the future, small coronary arteries might be categorized as a separate pathological subgroup.

\subsection{Limitation}

The observational nature of the study and the small sample size are among the most important limitations of the study. The small sample size influenced the power of our study, and choosing a larger population in future trials will have a more discriminating effect. Also, using more sophisticated tools (e.g. intracoronary imaging methods) for sizing the coronary arteries might increase the precision of the present evaluation.

\section{Acknowledgments}

We would like to thank the cardiac electrophysiology research center, Rajaie cardiovascular medical and research center staff for collaboration in preparing the manuscript.

\section{Footnote}

Funding/Support: This project was financially supported by the fund of Iran University of medical Science.

\section{References}

1. Eaker ED, Kronmal R, Kennedy JW, Davis K. Comparison of the longterm, postsurgical survival of women and men in the Coronary Artery Surgery Study (CASS). Am Heart J. 1989;117(1):71-81. [PubMed: 2643286].

2. Kelsey SF, James M, Holubkov AL, Holubkov R, Cowley MJ, Detre KM. Results of percutaneous transluminal coronary angioplasty in women. 1985-1986 National Heart, Lung, and Blood Institute's Coronary Angioplasty Registry. Circulation. 1993;87(3):720-7. [PubMed: 8443892].

3. Vaccarino V, Krumholz HM, Berkman LF, Horwitz RI. Sex differences in mortality after myocardial infarction. Is there evidence for an increased risk for women? Circulation. 1995;91(6):1861-71. [PubMed: 7882498]. 
Table 3. Multivariate Regression Test Results

\begin{tabular}{|c|c|c|c|c|}
\hline & Coronary Vessels & Standard Error & Beta & P Value \\
\hline \multirow{4}{*}{ Age } & LMCA & 0.008 & 0.01 & 0.8 \\
\hline & LAD & 0.007 & 0.002 & 0.9 \\
\hline & LCX & 0.009 & 0.07 & 0.4 \\
\hline & RCA & 0.008 & 0.17 & 0.1 \\
\hline \multirow{4}{*}{ Sex } & LMCA & 0.2 & 0.2 & 0.02 \\
\hline & LAD & 0.1 & 0.2 & 0.1 \\
\hline & LCX & 0.2 & 0.06 & 0.6 \\
\hline & RCA & 0.2 & 0.08 & 0.5 \\
\hline \multirow{4}{*}{ Dyslipidemia } & LMCA & 0.5 & 0.14 & 0.18 \\
\hline & LAD & 0.001 & 0.01 & 0.8 \\
\hline & LCX & 0.002 & -0.04 & 0.7 \\
\hline & RCA & 0.002 & 0.05 & 0.5 \\
\hline \multirow{4}{*}{ HTN } & LMCA & 0.1 & 0.08 & 0.4 \\
\hline & LAD & 0.1 & 0.08 & 0.4 \\
\hline & LCX & 0.1 & 0.09 & 0.4 \\
\hline & RCA & 0.1 & 0.1 & 0.3 \\
\hline \multirow{4}{*}{ DM } & LMCA & 0.2 & -0.22 & 0.09 \\
\hline & LAD & 0.1 & -0.072 & 0.5 \\
\hline & LCX & 0.2 & 0.002 & 0.9 \\
\hline & RCA & 0.2 & -0.2 & 0.08 \\
\hline \multirow{4}{*}{$\mathbf{C} / \mathbf{S}$} & LMCA & 0.2 & -0.1 & 0.4 \\
\hline & LAD & 0.2 & 0.04 & 0.7 \\
\hline & LCX & 0.2 & 0.01 & 0.9 \\
\hline & RCA & 0.2 & 0.1 & 0.3 \\
\hline \multirow{4}{*}{ FH } & LMCA & 0.2 & 0.08 & 0.4 \\
\hline & LAD & 0.1 & 0.17 & 0.09 \\
\hline & LCX & 0.2 & 0.08 & 0.4 \\
\hline & RCA & 0.2 & 0.1 & 0.2 \\
\hline
\end{tabular}

Abbreviations: C/S, cigar smoking; DLP, dyslipidemia; DM, diabetes mellitus; FH, family history; HTN, hypertension; LAD, left anterior descending; LCX, left circumflex artery; LMCA, left main coronary artery; RCA, right coronary artery.

4. Mintz GS, Popma JJ, Pichard AD, Kent KM, Salter LF, Chuang YC, et al. Intravascular ultrasound predictors of restenosis after percutaneous transcatheter coronary revascularization. J Am Coll Cardiol. 1996;27(7):1678-87. [PubMed: 8636553].

5. Fisher LD, Kennedy JW, Davis KB, Maynard C, Fritz JK, Kaiser G, et al. Association of sex, physical size, and operative mortality after coronary artery bypass in the Coronary Artery Surgery Study (CASS). J Thorac Cardiovasc Surg. 1982;84(3):334-41. [PubMed: 6981033].

6. Fuster V, Badimon L, Badimon JJ, Chesebro JH. The pathogenesis of coronary artery disease and the acute coronary syndromes (1). $N$ Engl J Med. 1992;326(4):242-50. doi: 10.1056/NEJM199201233260406. [PubMed: 1727977].

7. Burke AP, Farb A, Malcom GT, Liang YH, Smialek J, Virmani R. Coronary risk factors and plaque morphology in men with coronary disease who died suddenly. $N$ Engl J Med. 1997;336(18):1276-82. doi: 10.1056/NEJM199705013361802. [PubMed: 9113930].

8. Jeremias A, Spies C, Herity NA, Pomerantsev E, Yock PG, Fitzgerald PJ, et al. Coronary artery compliance and adaptive vessel remodelling in patients with stable and unstable coronary artery disease. Heart. 2000;84(3):314-9. [PubMed:10956298].

9. Dodge JJ, Brown BG, Bolson EL, Dodge HT. Lumen diameter of normal human coronary arteries. Influence of age, sex, anatomic variation, and left ventricular hypertrophy or dilation. Circulation. 1992;86(1):232-46. [PubMed: 1535570].

10. MacAlpin RN, Abbasi AS, Grollman JJ, Eber L. Human coronary artery size during life. A cinearteriographic study. Radiology. 1973;108(3):567-76. doi: 10.1148/108.3.567. [PubMed: 4269246].

11. Roberts CS, Roberts WC. Cross-sectional area of the proximal portions 
of the three major epicardial coronary arteries in 98 necropsy patients with different coronary events. Relationship to heart weight, age and sex. Circulation. 1980;62(5):953-9. [PubMed: 7418180].

12. Leung WH, Stadius ML, Alderman EL. Determinants of normal coronary artery dimensions in humans. Circulation. 1991;84(6):2294-306. [PubMed: 1959185].

13. Kornowski R, Lansky AJ, Mintz GS, Kent KM, Pichard AD, Satler LF, et al. Comparison of men versus women in cross-sectional area luminal narrowing, quantity of plaque, presence of calcium in plaque, and lumen location in coronary arteries by intravascular ultrasound in patients with stable angina pectoris. Am J Cardiol. 1997;79(12):1601-5. [PubMed: 9202348].

14. Sheifer SE, Canos MR, Weinfurt KP, Arora UK, Mendelsohn FO, Gersh BJ, et al. Sex differences in coronary artery size assessed by intravascular ultrasound. Am Heart J. 2000;139(4):649-53. [PubMed: 10740147].

15. Guerra OR, Janowitz WR, Agatston AS, Mantelle LL, Viamonte MJ. Coronary artery diameter and coronary risk factors: a study with ultrafast computed tomography. Am Heart J. 1993;126(3 Pt 1):600-6. [PubMed: 8362715].
16. Hamirani YS, Nasir K, Avanes E, Kadakia J, Budoff MJ. Coronary artery diameter related to calcium scores and coronary risk factors as measured with multidetector computed tomography: a substudy of the ACCURACY trial. Tex Heart Inst J. 2013;40(3):261-7. [PubMed: 23914015]

17. McGill HJ, McMahan CA, Tracy RE, Oalmann MC, Cornhill JF, Herderick EE, et al. Relation of a postmortem renal index of hypertension to atherosclerosis and coronary artery size in young men and women. Pathobiological Determinants of Atherosclerosis in Youth (PDAY) Research Group. Arterioscler Thromb Vasc Biol. 1998;18(7):110818. [PubMed: 9672071].

18. Morgan KP, Kapur A, Beatt KJ.Anatomy of coronary disease in diabetic patients: an explanation for poorer outcomes after percutaneous coronary intervention and potential target for intervention. Heart. 2004;90(7):732-8. doi: 10.1136/hrt.2003.021014. [PubMed: 15201238].

19. Mosseri M, Nahir M, Rozenman Y, Lotan C, Admon D, Raz I, et al. Diffuse narrowing of coronary arteries in diabetic patients: the earliest phase of coronary artery disease. Cardiology. 1998;89(2):103-10. [PubMed: 9524010]. 